TITLE:

\title{
Initial value represented propagator for semiquantal squeezed state wave packet
}

$\operatorname{AUTHOR}(S)$ :

Ando, Koji

CITATION:

Ando, Koji. Initial value represented propagator for semiquantal

squeezed state wave packet. Chemical Physics Letters 2014, 591: 179-

184

ISSUE DATE:

2014-01

URL:

http://hdl.handle.net/2433/180113

RIGHT:

(C) 2013 Elsevier B.V.; This is not the published version. Please cite only the published version.; この論文は出版社版でありません。引用の際に は出版社版をご確認ご利用ください。 


\title{
Initial value represented propagator for semiquantal squeezed state wave packet
}

\author{
Koji Ando* \\ Department of Chemistry, Graduate School of Science, Kyoto University, Sakyo-ku, Kyoto 606-8502, Japan
}

\begin{abstract}
The initial-value representation (IVR) of semiclassical propagator for the semiquantal (SQ) squeezed-state wave packet (WP) is examined. The SQ IVR is derived naturally from the coherent-state path-integral theory, in which similarity and difference from the conventional semiclassical methods are transparent. The accuracy of SQ IVR is assessed numerically on assorted schemes of treating the WP width. It is found to yield accurate wave function propagation when the WP width is optimized initially on the SQ potential and its dynamical motion is frozen or damped.
\end{abstract}

\section{INTRODUCTION}

Realistic simulation of quantum mechanical (QM) molecular dynamics (MD) is of fundamental and practical importance open to current active investigations. Full QM calculation has been hindered by exponential increase of computational cost along the number of degrees of freedom due to the inherent QM non-locality. On the other hand, local nature of classical mechanical equations of motion (EOM) allows simulations of larger systems, so that the computational bottleneck is around the evaluation of potential energies rather than the time propagation: with proper treatment of the former, well over thousands of atoms can be handled nowadays.

To fill the gap between QM and classical limits, various semiclassical and related methods have been developed, including the initial-value representation (IVR) of propagator $^{1-3}$ with the Herman-Kluk (HK) prefactor ${ }^{4-6}$, integral-expression $(\mathrm{IE})^{7}$, and related developments ${ }^{8-12}$, coupled coherentstate $^{13}$, multi-configuration time-dependent Hartree ${ }^{14}$, actiondecomposed function ${ }^{15}$, and Bohmian approaches ${ }^{16}$. Each has its own pros and cons in details, but most of them yield accurate wave function propagation for small systems, except for the challenging cases of deep-tunneling. Applications to large and complex molecular systems are yet scarce, while steady progress is observed these years.

Besides these wave function approaches, methods based on the imaginary-time path-integral (PI) theory have been developed and applied to condensed phase simulations with realistic molecular models ${ }^{17-22}$. Their computational efficiency is secured by limiting the QM effects to the zero-point delocalization, whose adequacy seems to be supported by the anticipated decoherence in condensed phases. The effects pertaining to the QM phase, such as deep-tunneling and interference, are mostly suppressed by coupling to many degrees of freedom, in contrast with the zero-point effects that mainly originate from the uncertainty.

Similar parts of QM effects are covered also by the semiquantal (SQ) wave packet (WP) MD method ${ }^{23-28}$, which

\footnotetext{
*Fax: +81757534020

E-mail address: ando@kuchem.kyoto-u.ac.jp

Chemical Physics Letters 591 (2014) 179-184.

http://dx.doi.org/10.1016/j.cplett.2013.11.032
}

serves an intermediate between the imaginary-time PI and the wave function approaches by taking account of the zeropoint fluctuation and dynamic WP broadening in real-time and real-space. It is thus capable of describing WP delocalization over low-barrier hydrogen-bonds, under-barrier shallow tunneling in nearly-adiabatic proton transfers, and geometric isotope effects in realistic molecular systems ${ }^{23-25}$. It is also free from the problem of zero-point energy leakage ${ }^{21,22}$. For instance, the critical barrier height for adiabatic proton transfer is $\sim 2 \mathrm{kcal} / \mathrm{mol}^{29}$, which hinders the ordinary classical dynamics with the thermal energy of $300 \mathrm{~K} \simeq 0.6 \mathrm{kcal} / \mathrm{mol}$ but can be properly handled by the WP delocalization.

The SQ WP scheme is similar to the thawed Gaussian approximation $^{30}$ (TGA) in allowing dynamical motion of the WP width, but is distinguished by the canonical Hamiltonian form of EOM for both the WP center and width variables that yields stable symplectic propagation, as has been demonstrated in the simulation of liquid water ${ }^{26-28}$. The EOM are derived via the time-dependent variational principle in which the action integral is extremized. This is related to the stationary-phase of propagator in the coherent-state (CS) PI representation $^{31-33}$. In this regard, the SQ WP and the IVR propagator will be naturally connected, which is awaiting precise formulation and quantitative evaluation.

The purpose of this Letter is to explore such IVR scheme to be combined with the SQ WP framework. In the current SQ WP MD simulation, the WP remains to be a squeezed-state Gaussian WP during the propagation. This seems an adequate model for equilibrium properties such as structural distribution and incoherent processes such as diffusion in condensed phases, for the same reason (i.e., decoherence) as for the success of imaginary-time PI methods. Nevertheless, it is desired to have an option to boost the accuracy when needed, to describe more complicated wave functions, in a consistent and seamless manner to take the advantages of SQ scheme.

After outlining the SQ WP and the CS PI theories, we describe the IVR propagator for SQ WP in Sec. II. Numerical calculations are performed in Sec. III on assorted schemes of treating the WP width. Section IV concludes with a summary and outlook. 


\section{THEORY}

\section{A. Semiquantal Squeezed State Wave Packet}

Here we summarize the SQ WP theory $23,34,35$ in onedimension. Extension to many-dimension is straightforward, but involves complication around correlations among degrees of freedom, ${ }^{27}$ which shall be addressed elsewhere.

The form of squeezed-state WP we consider is thus

$\phi_{\mathrm{sq}}(x, t)=\left(2 \pi \rho_{t}^{2}\right)^{-1 / 4} \exp \left[-\gamma_{t}\left(x-q_{t}\right)^{2}+\frac{i}{\hbar} p_{t}\left(x-q_{t}\right)\right]$,

specified by the time-dependent variables $q_{t}, p_{t}$, and $\gamma_{t}$. As in the ordinary CS WP, $q_{t}$ and $p_{t}$ represent the WP center and its momentum. $\gamma_{t}$ is a complex variable of a form

$$
\gamma_{t}=\frac{1}{4 \rho_{t}^{2}}-\frac{i}{2 \hbar} \frac{\pi_{t}}{\rho_{t}}
$$

in which $\rho_{t}$ represents the WP width. $\pi_{t}$ is the momentum conjugate to $\rho_{t}$, as will be seen in the EOM (8) derived by the time-dependent variational principle.

With the Hamiltonian operator for a mass $m$ under potential $V$,

$$
\hat{H}=-\frac{\hbar^{2}}{2 m} \frac{\partial^{2}}{\partial x^{2}}+V(x)
$$

the action integral $S_{\mathrm{sq}}$ is defined by

$$
S_{\mathrm{sq}}=\int_{0}^{t} L_{\mathrm{sq}} d t^{\prime}=\int_{0}^{t} d t^{\prime}\left\langle\phi_{\mathrm{sq}}\left(t^{\prime}\right)\left|i \hbar \frac{\partial}{\partial t^{\prime}}-\hat{H}\right| \phi_{\mathrm{sq}}\left(t^{\prime}\right)\right\rangle,
$$

which also defines the Lagrangian $L_{\mathrm{sq}}$. For Eqs. (1) and (3) we find

$$
L_{\mathrm{sq}}=p \dot{q}+\frac{1}{2}(\pi \dot{\rho}-\rho \dot{\pi})-H_{\mathrm{sq}}
$$

with

$$
H_{\mathrm{sq}}=\langle\hat{H}\rangle=\frac{p^{2}}{2 m}+\frac{\pi^{2}}{2 m}+\frac{\hbar^{2}}{8 m \rho^{2}}+\langle V\rangle,
$$

in which $\langle\cdots\rangle$ denotes the expectation value with respect to $\phi_{\text {sq }}(x, t)$.

It is seen in Eq. (5) that the variational condition, $\delta S_{\mathrm{sq}} / \delta q=\delta S_{\mathrm{sq}} / \delta \rho=0$, yields the EOM of canonical Hamilton form,

$$
\begin{array}{ll}
\dot{q}=\frac{\partial H_{\mathrm{sq}}}{\partial p}, & \dot{p}=-\frac{\partial H_{\mathrm{sq}}}{\partial q}, \\
\dot{\rho}=\frac{\partial H_{\mathrm{sq}}}{\partial \pi}, & \dot{\pi}=-\frac{\partial H_{\mathrm{sq}}}{\partial \rho} .
\end{array}
$$

We thus regard $(q, p)$ and $(\rho, \pi)$ as conjugate coordinate and momentum pairs, and study their dynamics on the potential in an extended phase-space $(q, \rho, p, \pi)$. (Incidentally, this aspect is shared by the approaches based on expectation-values of moments ${ }^{36}$ or cumulants ${ }^{37}$.) The last two terms of Eq. defines the SQ potential

$$
V_{\mathrm{sq}}(q, \rho)=\frac{\hbar^{2}}{8 m \rho^{2}}+\langle V\rangle(q, \rho) .
$$

In general cases where the evaluation of $\langle V\rangle$ is not trivial, we expand $V(x)$ around $\langle x\rangle=q$ to find

$$
\langle V\rangle=V(q)+\frac{1}{2} V^{(2)}(q) \rho^{2}+\frac{1}{8} V^{(4)}(q) \rho^{4}+\cdots,
$$

in which $V^{(n)}$ denotes the $n$-th derivative. An application to the Lippincott-Schroeder model of hydrogen-bond potential has confirmed that the expansion up to the fourth-order yields reasonably accurate wave functions and energies ${ }^{25}$. On the other hand, direct evaluation of $\langle V\rangle$ will be possible in combination with the standard ab initio molecular orbital calculations by straightforward Gaussian integrations, similar to that in the combined nuclear and molecular orbital methods ${ }^{38}$. The same will apply to the electron WP method with valence-bond coupling ${ }^{39,40}$.

\section{B. Coherent-State Path-Integral}

We introduce the CS $\left|q_{t}, p_{t} ; \gamma_{t}\right\rangle$ so that $\phi_{\mathrm{sq}}(x, t)$ of Eq. (1) is given by

$$
\phi_{\mathrm{sq}}(x, t)=\left\langle x \mid q_{t}, p_{t} ; \gamma_{t}\right\rangle \text {, }
$$

which forms an overcomplete basis,

$$
\iint \frac{d q_{t} d p_{t}}{2 \pi \hbar}\left|q_{t}, p_{t} ; \gamma_{t}\right\rangle\left\langle q_{t}, p_{t} ; \gamma_{t}\right|=1
$$

Note that $\gamma_{t}$ is arbitrary here.

Using this identity, the QM propagator is represented by the CS PI,

$$
\begin{aligned}
& K\left(x, t ; x^{\prime}, 0\right)=\left\langle x|\exp [-i \hat{H} t / \hbar]| x^{\prime}\right\rangle \\
& =\iiint \int \frac{d q_{t} d p_{t}}{2 \pi \hbar} \frac{d q_{0} d p_{0}}{2 \pi \hbar}\left\langle x \mid q_{t}, p_{t} ; \gamma_{t}\right\rangle\left\langle q_{0}, p_{0} ; \gamma_{0} \mid x^{\prime}\right\rangle \\
& \quad \times \iint \mathcal{D}[q(t) p(t)] \exp \left[\frac{i}{\hbar} S_{\mathrm{sq}}[q(t), p(t)]\right],
\end{aligned}
$$

in which $\iint \mathcal{D}[q(t) p(t)]$ denotes the functional integration. The action $S_{\mathrm{sq}}$ in the PI is identical to that in Eq. (4). Therefore, the stationary-phase of PI yields the EOM (7) of $q$ and $p$. However, the EOM (8) of $\rho$ and $\pi$ are not derived this way because of the arbitrariness of $\gamma_{t}$ in Eq. (12). In this sense, they are subject to options to be explored on various schemes, as will be described in Sec. III

\section{IVR Propagator}

To evaluate the PI in Eq. (13) by the steepest-descent method, we follow Ref. ${ }^{11}$ which has explicitly dealt with a 
general form of time-dependent WP width. It is thus straightforward to find the IVR propagator for the present SQ WP,

$$
\begin{aligned}
& K_{\mathrm{sq}}^{\mathrm{sd}}\left(x, t ; x^{\prime}, 0\right)=\iint \frac{d q_{0} d p_{0}}{2 \pi \hbar}\left\langle x \mid q_{t}, p_{t} ; \gamma_{t}\right\rangle C\left(q_{0}, p_{0}, t\right) \\
& \times \exp \left[\frac{i}{\hbar} S_{\mathrm{sq}}^{\mathrm{sd}}\left(q_{0}, p_{0}, t\right)\right]\left\langle q_{0}, p_{0} ; \gamma_{0} \mid x^{\prime}\right\rangle
\end{aligned}
$$

in which the action integral is given by

$S_{\mathrm{sq}}^{\mathrm{sd}}\left(q_{0}, p_{0}, t\right)=\int_{0}^{t} d \tau\left(p_{\tau} \dot{q}_{\tau}-\frac{p_{\tau}^{2}}{2 m}-\langle V\rangle_{\tau}+\frac{1}{2} \frac{\partial^{2}\langle V\rangle_{\tau}}{\partial q_{\tau}^{2}} \rho_{\tau}^{2}\right)$

with $\langle V\rangle_{\tau}=\langle V\rangle\left(q_{\tau}, \rho_{\tau}\right)$. This originates from the SQ action $S_{\text {sq }}$ in Eq. (13), but the terms related to the WP width, $\pi^{2} / 2 m$ and $\hbar^{2} / 8 m \rho^{2}$, have been crossed out by those coming from the steepest-descent evaluation. This applies independently of whether their dynamics follow the EOM (8) or not, which is another ground for our testing assorted schemes for the WP width in Sec. III.

We see the integrand of $S_{\mathrm{sq}}^{\mathrm{sd}}$ is similar to that of classical action,

$$
S_{\mathrm{cl}}\left(q_{0}, p_{0}, t\right)=\int_{0}^{t} d \tau\left(p_{\tau} \dot{q}_{\tau}-\frac{p_{\tau}^{2}}{2 m}-V\left(q_{\tau}\right)\right)
$$

as the leading second-order term in the expansion of Eq. (10) is eliminated in Eq. (15). However, the terms that appear common in $S_{\mathrm{sq}}^{\mathrm{sd}}$ and $S_{\mathrm{cl}}$ are practically different since the potentials that generate the trajectories $\left(q_{\tau}, p_{\tau}\right)$ are uncommon: the full SQ potential $V_{\mathrm{sq}}$ of Eq. (9) for the former versus the bare classical potential $V(q)$ for the latter. The use of $S_{\mathrm{cl}}$ in the original HK frozen Gaussian (FG) scheme ${ }^{4,5}$ and Kay's $\mathrm{IE}^{7}$ was by design. Their deviation from the CS PI framework would depend on specific potential functions. As seen here, this aspect is transparent in the SQ framework.

The prefactor $C\left(q_{0}, p_{0}, t\right)$ in Eq. (14) is given by

$$
\begin{gathered}
C\left(q_{0}, p_{0}, t\right)=\left(\operatorname{Re} \gamma_{t} \operatorname{Re} \gamma_{0}\right)^{-1 / 4} \\
\times\left[\frac{1}{2}\left(\gamma_{t} \frac{\partial q_{t}}{\partial q_{0}}+\gamma_{0}^{*} \frac{\partial p_{t}}{\partial p_{0}}-2 i \hbar \gamma_{t} \gamma_{0}^{*} \frac{\partial q_{t}}{\partial p_{0}}+\frac{i}{2 \hbar} \frac{\partial p_{t}}{\partial q_{0}}\right)\right]^{1 / 2},
\end{gathered}
$$

which is identical to that derived by $\mathrm{Kay}^{7}$ in the IE formulation which generalized the HK FG formula to take account of the WP width parameters $\gamma_{0}$ and $\gamma_{t}$ at both ends of propagation. The original HK FG prefactor is retrieved by setting $\gamma_{t}=\gamma_{0}$ and nullifying their imaginary part.

The partial derivatives (i.e., monodromy matrix) in Eq. (17) propagate according to their EOM, which requires second derivatives of the potential. This is similar between the SQ and HK FG cases $^{5}$; the difference is only in the form of the potential, $V_{\text {ext }}$ or the bare classical $V$.

In summary, the working equations of SQ IVR propagator are (14) and (15), in which the trajectories of WP center $\left(q_{t}, p_{t}\right)$ are generated from the EOM (7). On the other hand, the WP width $\left(\rho_{t}, \pi_{t}\right)$ in this framework are not required to follow the EOM (8) but are open for options in practice, which we will examine in Sec. III.

\section{NUMERICAL APPLICATIONS}

\section{A. Treatment of Wave Packet Width}

The way to treat WP width has been an issue in the HK FG scheme. Some optimized it in various ways ${ }^{41-43}$, and others attempted to refine the $\mathrm{TGA}^{3,9}$. In this regard, the extended potential concept in the canonical SQ framework described in Sec. II A may be useful to set a well-defined WP width in a simple and comprehensive manner. We thus consider the following schemes:

1. The SQ scheme with a frozen WP width $\gamma_{0}=\gamma_{t}=1 / 4 \rho^{2}$ such that the value of $\rho$ gives the global minimum of $V_{\mathrm{sq}}(q, \rho)$. This is denoted ' $\mathrm{SQ}(\mathrm{fix})$ '.

2. The SQ scheme with time-dependent $\gamma_{\tau}$ composed via Eq. (2) of $\rho_{\tau}$ and $\pi_{\tau}$ that follow the EOM (8). The initial condition $\rho_{0}$ is set in the same way as in SQ(fix) above, whereas $\pi_{0}$ is set to be zero. This scheme with undamped WP width is denoted 'SQ(udmp)'.

3. Similar to SQ(udmp) above, but with an augmented friction term to damp the motions of $\rho_{\tau}$ and $\pi_{\tau}$ with relaxation time $\tau_{r}$. That is, the second of Eq. (8) is modified to $\dot{\pi}=-\partial H_{\mathrm{sq}} / \partial \rho-\pi / \tau_{r}$. The initial condition $\left(\rho_{0}, \pi_{0}\right)$ is set in the same way as in SQ(udmp) above. This is denoted 'SQ(dmp)'.

4. The original HK FG scheme with the propagating WP having the same width as the initial wave function $\psi(x, 0)$, namely, we set $\gamma_{0}=\gamma_{t}=\gamma_{i}$ (see Eq. (20) for the definition of $\gamma_{i}$ ). We denote this 'HK(orig)'.

5. A hybrid of HK FG and SQ(fix), in which we set $\gamma_{0}=$ $\gamma_{t}=1 / 4 \rho^{2}$ in the same way as in SQ(fix). The difference from $\mathrm{SQ}(\mathrm{fix})$ is that the classical Hamiltonian and action, $H_{\mathrm{cl}}$ and $S_{\mathrm{cl}}$, are employed. We denote this 'HK(opt 0 )'.

6. Similar to HK(opt 0 ) but the value of $\rho$ is optimized only at the end time $t$ to set $\gamma_{t}=1 / 4 \rho^{2}$ such that $V_{\mathrm{sq}}\left(q_{t}, \rho\right)$ is minimized locally along $\rho$ at the end position $q_{t}$. The initial width is set $\gamma_{0}=\gamma_{i}$, similarly to $\mathrm{HK}$ (orig). In this scheme, the generalized prefactor of Eq. (17) for different $\gamma_{t}$ and $\gamma_{0}$ is essential. We denote this ' $\mathrm{HKK}(\mathrm{opt} \mathrm{t})$ ' after Herman-Kluk-Kay.

7. A combination of $\mathrm{HK}$ (opt 0 ) and $\mathrm{HKK}$ (opt $\mathrm{t}$ ), where $\gamma_{0}$ and $\gamma_{t}$ are determined as in $\mathrm{HK}$ (opt 0 ) and HKK(opt t), respectively. We denote this 'HKK(opt $0 \mathrm{t}$ )'.

These schemes are summarized in Table 1. Additional points to note are:

(i) HK(orig) would be the simplest choice and might also appear natural. It has been thus employed previously ${ }^{9}$ (but see Footnote 15 in this reference) for the same potential function as we study in Sec. III B.

(ii) A novel aspect in $\mathrm{HK}$ (opt 0 ) and $\mathrm{HKK}$ (opt t ) compared to the conventional HK scheme is to exploit the SQ potential $V_{\mathrm{sq}}(q, \rho)$ to set the WP width parameters $\gamma_{0}$ and $\gamma_{t}$ in a simple and well-defined way. The global optimization of the WP width $\rho$ on $V_{\mathrm{sq}}(q, \rho)$ has been demonstrated 
Table 1. A summary of seven schemes. See Sec. III A for details.

\begin{tabular}{llc}
\hline Scheme & Wave packet width & Action \\
\hline SQ(fix) & $\gamma_{0}=\gamma_{t}=1 / 4 \rho^{2}$ & $S_{\mathrm{sq}}^{\text {sd }}$ \\
SQ(udmp) & $\gamma_{\tau}=1 / 4 \rho_{\tau}^{2}+i \pi_{\tau} / 2 \hbar \rho_{\tau}$ & $S_{\mathrm{sq}}^{\text {sd }}$ \\
SQ(dmp) & $\gamma_{\tau}$ of SQ(udmp) plus damping & $S_{\mathrm{sq}}^{\mathrm{sd}}$ \\
HK(orig) & $\gamma_{0}=\gamma_{t}=\gamma_{i}$ & $S_{\mathrm{cl}}$ \\
HK(opt 0) & $\gamma_{0}=\gamma_{t}=1 / 4 \rho^{2}$ & $S_{\mathrm{cl}}$ \\
HKK(opt t) & $\gamma_{0}=\gamma_{i}, \gamma_{t}=1 / 4 \rho_{t}^{2}$ & $S_{\mathrm{cl}}$ \\
HKK(opt 0t) & $\gamma_{0}=1 / 4 \rho^{2}, \gamma_{t}=1 / 4 \rho_{t}^{2}$ & $S_{\mathrm{cl}}$ \\
\hline
\end{tabular}

to yield optimal approximation to the ground state wave function for bound potentials $\mathrm{s}^{23,25,44}$. On the other hand, the optimization of $\gamma_{t}$ in $\operatorname{HKK}$ (opt t) and HKK(opt 0t) is carried out locally at $q_{t}$ along $\rho$ on $V_{\mathrm{sq}}\left(q_{t}, \rho\right)$. [We did not carry out corresponding local optimization at $q_{0}$ for $\gamma_{0}$ because they are mutually dependent in the Monte Carlo (MC) sampling (see Eq. (24))].

(iii) In HKK(opt t), $\gamma_{t}$ does not follow any EOM but is optimized only at each end time $t$. Note that the value of $\gamma_{\tau}$ in the intermediate time $0<\tau<t$ is irrelevant in the HK FG scheme since only the classical Hamiltonian and action are employed for the propagation.

(iv) The initial phase factor of the propagating WP is set by $p_{0}$ and $\pi_{0}$ (see Eq. (1)): while the former is determined by the MC sampling as specified in Sec. III B (see also Eq. (24)), the latter is set arbitrarily to zero in all cases.

\section{B. Computational Details}

The potential function we examine here is the ordinary Morse potential,

$$
V(q)=V_{0}\left(1-\mathrm{e}^{-\lambda q}\right)^{2},
$$

with the parameters $\lambda=0.08$ and $V_{0}=30$. These and the mass $m=1$ defines the scaled unit time length. It is straightforward to find the SQ potential Eq. (9) for Eq. (18),

$V_{\mathrm{sq}}(q, \rho)=\frac{\hbar^{2}}{8 m \rho^{2}}+V_{0}\left(1-2 \mathrm{e}^{-\lambda q+\lambda^{2} \rho^{2} / 2}+\mathrm{e}^{-2 \lambda q+2 \lambda^{2} \rho^{2}}\right)$.

The initial wave function $\psi(x, 0)$ is set to the same form as Eq. (1),

$$
\psi(x, 0)=\langle x \mid \psi(0)\rangle=\left\langle x \mid q_{i}, p_{i} ; \gamma_{i}\right\rangle,
$$

with $q_{i}=p_{i}=0$ and $\gamma_{i}=6$, where we have introduced the subscript $i$ to denote the initial wave function. All the parameters, $\lambda, V_{0}, m, q_{i}, p_{i}$, and $\gamma_{i}$, are taken from Ref. ${ }^{9}$. Note the distinction between $\gamma_{i}$ for the initial wave function and $\gamma_{0}$ for the propagating WP, which is the key to the comparison between HK(orig) and HK(opt 0).

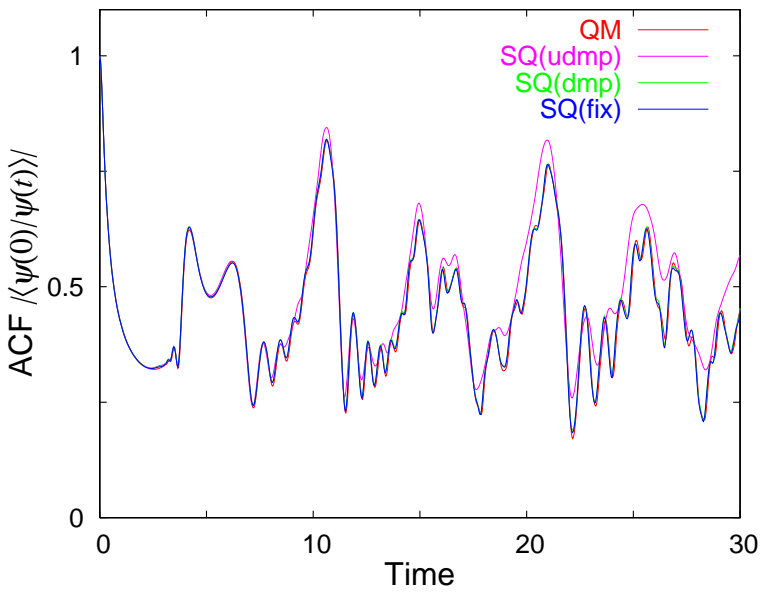

FIG. 1: Absolute auto-correlation function of the time-dependent wave function, Eq. (21), for SQ(fix), SQ(udmp), and SQ(dmp) that employ the SQ action $S_{\mathrm{sq}}^{\text {sd }}$ (see Sec. III A and Table 1 for details), compared with QM reference. SQ(fix) and SQ(dmp) overlap with $\mathrm{QM}$ such that they are indistinguishable in the figure.

For the reference QM calculation, we employ a real-space finite spatial differentiation and Cayley's hybrid form of implicit and explicit schemes for unitary time evolution. ${ }^{45}$ Convergence and unitarity of time propagation have been confirmed with the grid length $\Delta x=0.05$ and the time step $\Delta t=6 \times 10^{-5}$.

The trajectories of $(q, p)$ and $(\rho, \pi)$ conjugate pairs are propagated by Suzuki's symplectic fourth-order fractal decomposition scheme ${ }^{46}$ Conservation of energy has been confirmed with the time step $\Delta t=8 \times 10^{-3}$. The initial conditions $\left(q_{0}, p_{0}\right)$ are generated by the $\mathrm{MC}$ sampling technique to reproduce the initial wave function $\psi(x, 0)$ (see Eq. (24) $)^{5}$. The number of sampled trajectories $N_{\mathrm{MC}}$ is up to $6400 .{ }^{7}$ The branch of square-root in $C\left(q_{0}, p_{0}, t\right)$ is taken to be continuous along $t$ by monitoring its phase alteration in succeeding time steps. Conservation of the norm of wave function $\psi(x, t)$ is checked in Fig. 3, before it is normalized for computing the auto-correlation functions (ACF) in Figs 1 and 2 and the probability density in Fig. 4. The relaxation time in $\mathrm{SQ}(\mathrm{dmp})$ is set $\tau_{r}=0.2$.

\section{Results}

Figure 1 shows the absolute value of ACF of the wave function

$$
c(t)=|\langle\psi(0) \mid \psi(t)\rangle|
$$

calculated from the IVR propagator,

$$
\psi(x, t)=\int d x^{\prime} K_{\mathrm{sq}}^{\mathrm{sd}}\left(x, t ; x^{\prime}, 0\right) \psi\left(x^{\prime}, 0\right),
$$

with the schemes that employ $S_{\mathrm{sq}}^{\mathrm{sd}}$. SQ(fix) and SQ(dmp) are almost indistinguishable from the QM reference in the fig- 


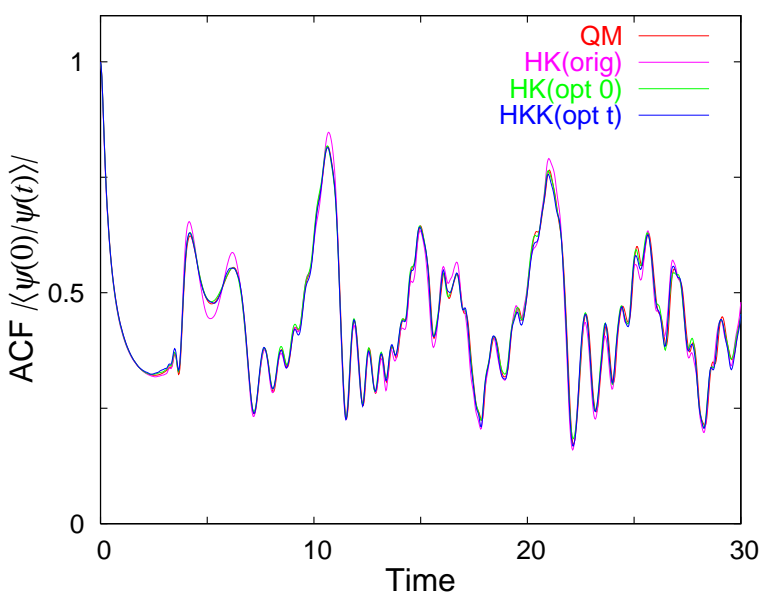

FIG. 2: Same as Fig. 1 but for HK(orig), HK(opt 0), and HKK(opt t) that employ the classical action $S_{\mathrm{cl}}$. HK(opt 0) and HKK(opt t) overlap with QM such that they are indistinguishable in the figure.

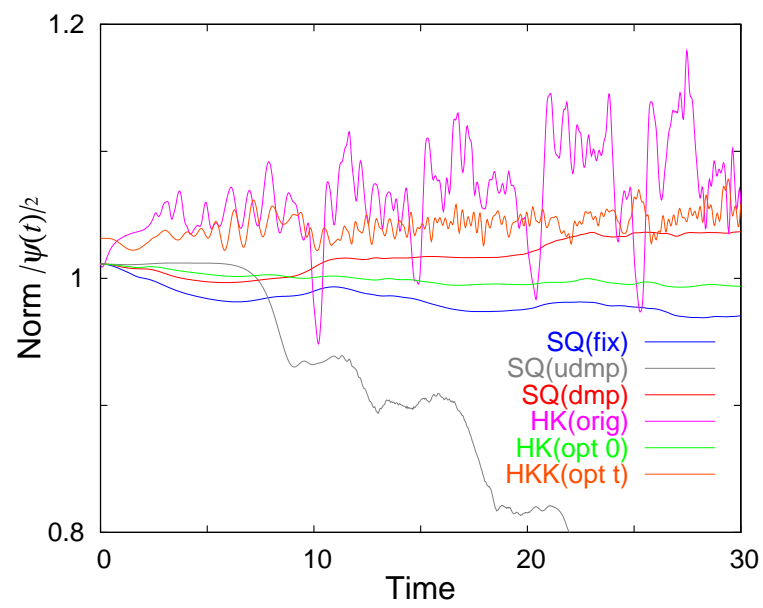

FIG. 3: Norm of wave function $|\psi(t)|^{2}$ for assorted schemes of treating the WP width, as explained in Sec. III A and Table 1.

ure. SQ(udmp) performs well in short time but gradually deteriorate in longer time. Figure 2 shows the ACFs from the schemes with $S_{\mathrm{cl}}$. Similarly to $\mathrm{SQ}($ fix) and SQ(dmp) in Fig. 1, HK(opt 0) and HKK(opt t) are almost indistinguishable from QM. HKK(opt 0t) was also found to overlap with QM as well, and was thus omitted from the figure. The finding that $\mathrm{HK}$ (opt 0 ) and $\mathrm{SQ}$ (fix) give equally good results implies that their differences in the Hamiltonian, $H_{\mathrm{cl}}$ and $H_{\mathrm{sq}}$, and in the action integral, $S_{\mathrm{cl}}$ and $S_{\mathrm{sq}}^{\mathrm{sd}}$, are not critical for this system. On the other hand, HK(orig) shows notable deviation from QM throughout the time range. This is obviously associated with the choice of the WP width in the FG scheme. We shall come back to this after going through the other results.

Another measure of accuracy is the norm of wave function $|\psi(t)|^{2}$ displayed in Fig. 3. The norm stays close to unity in $\mathrm{HK}$ (opt 0), SQ(fix), and SQ(dmp), whereas devia-

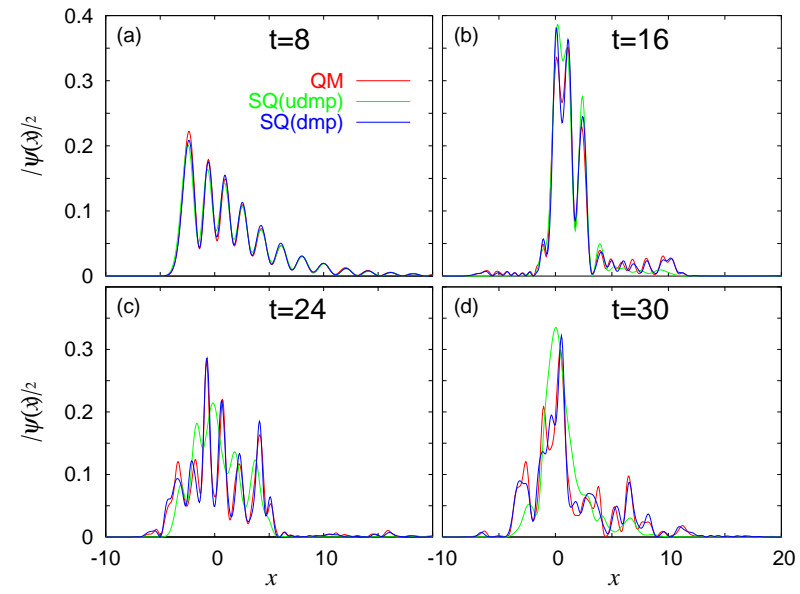

FIG. 4: Probability density $|\psi(x)|^{2}$ from SQ(udmp), SQ(dmp), and $\mathrm{QM}$ reference at four selected times. Results for the other schemes, SQ(fix), HK(opt 0), HKK(opt t), and HKK(opt 0t), have been omitted from the figure as they show better or comparable agreement with QM than SQ(dmp) does.

tion from unity is apparent in $\mathrm{SQ}(\mathrm{udmp}$ ) and $\mathrm{HK}$ (orig). These are consistent with the results in Figs. 1 and 2. The HK FG scheme has been shown to be unitary in the stationary-phase approximation $^{6}$, but in practice it is known to depend notably on numerical settings. The unitarity of SQ scheme is unknown at present, but would be presumed to depend similarly on numerics.

Figure 4 displays the probability density $|\psi(x)|^{2}$ at $t=8$, 16, 24, and 30, for $\mathrm{SQ}(\mathrm{udmp})$ and $\mathrm{SQ}(\mathrm{dmp})$ compared to $\mathrm{QM}$ reference. Although not included in the figure, SQ(fix), $\mathrm{HK}$ (opt 0), HKK(opt t), and HKK(opt 0t) are found to show better or comparable agreement with QM than $\mathrm{SQ}(\mathrm{dmp})$ does, as suggested from their results in Figs. 1-3. We see in Fig. 4 that even the worst results by SQ(udmp) were able to capture essential features of the distribution.

We have also checked the convergence along the number of MC trajectories $N_{\mathrm{MC}}$. Figure 5 shows the root-mean-squares deviation of the ACF $c(t)$

$$
\mathrm{rms}=\sqrt{\frac{1}{T} \int_{0}^{T}\left(c(t)-c_{\mathrm{QM}}(t)\right)^{2} d t}
$$

from the $\mathrm{QM}$ reference $c_{\mathrm{QM}}(t)$. The integration time is set to be $T=30$. We see that SQ(fix), SQ(dmp), and HK(opt 0 ) show comparative quality and almost converge at $N_{\mathrm{MC}}=$ 3200. Although not shown in the figure, similar convergence was found also for HKK(opt t) and HKK(opt 0t). The relative accuracy among the schemes basically follows that of the norm shown in Fig. 3. The problems in HK(orig) and SQ(udmp) are discussed next.

The FG width parameter $\gamma_{0}=\gamma_{i}=6$ used in HK(orig) is much larger than $\gamma_{0}=1 / 4 \rho^{2}=0.313$ found optimal in $\mathrm{HK}$ (opt 0$)$. The MC sampling of initial condition $\left(q_{0}, p_{0}\right)$ is 


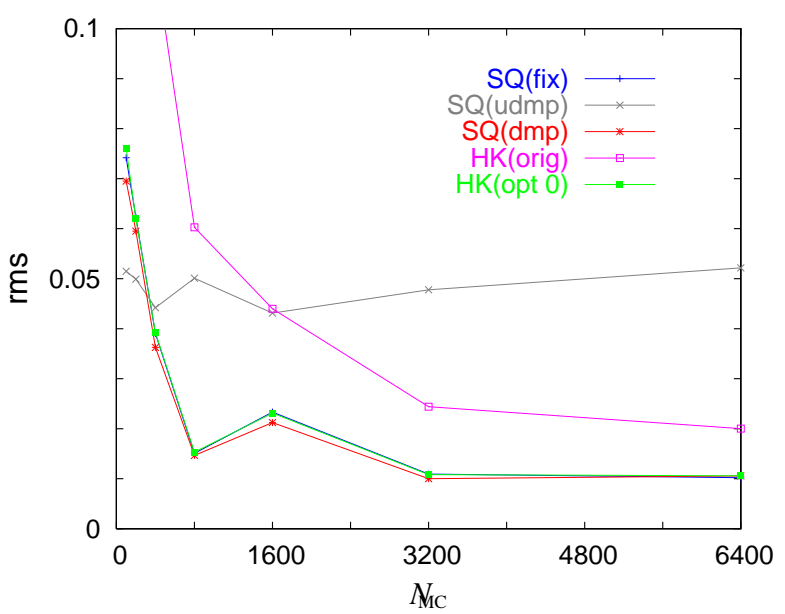

FIG. 5: Convergence along the number of Monte Carlo samplings $N_{\text {MC }}$ measured by the root-mean-squares deviation of the autocorrelation function from the QM reference, Eq. (23).

weighted according to the overlap factor

$$
\begin{gathered}
\left\langle q_{0}, p_{0} ; \gamma_{0} \mid \psi(0)\right\rangle=\left\langle q_{0}, p_{0} ; \gamma_{0} \mid q_{i}, p_{i} ; \gamma_{i}\right\rangle \\
=\sqrt{\frac{2 \sqrt{\gamma_{i} \gamma_{0}}}{\gamma_{i}+\gamma_{0}}} \exp \left[-\frac{\gamma_{i} \gamma_{0}}{\gamma_{i}+\gamma_{0}}\left(q_{0}-q_{i}\right)^{2}-\frac{\left(p_{0}-p_{i}\right)^{2}}{4 \hbar^{2}\left(\gamma_{i}+\gamma_{0}\right)}\right. \\
\left.\quad+\frac{i}{\hbar} \frac{\gamma_{i} p_{0}+\gamma_{0} p_{i}}{\gamma_{i}+\gamma_{0}}\left(q_{0}-q_{i}\right)\right]
\end{gathered}
$$

Thus, $q_{0}$ and $p_{0}$ are sampled in the normal Gaussian distributions scaled by the factors $\gamma_{i} \gamma_{0} /\left(\gamma_{i}+\gamma_{0}\right)$ and $1 / 4 \hbar^{2}\left(\gamma_{i}+\gamma_{0}\right)$, respectively. This indicates that the overestimation of $\gamma_{0}$ results in too broad distribution of the initial momentum $p_{0}$, which shall be the reason for the slow convergence of $\mathrm{HK}$ (orig).

The inferior accuracy of SQ(udmp) and its improvement by $\mathrm{SQ}(\mathrm{dmp})$ imply that the dynamical motion of WP width is superfluous in the current IVR framework where the WP width is arbitrary. In this regard, another remedy for SQ(udmp) might be found by deriving an alternative IVR scheme which will involve reexamination of the identity relation in place of Eq. (12) for the squeezed (two-photon coherent) state ${ }^{47}$ and significant modification of the prefactor Eq. (17).

\section{CONCLUSION}

The findings of this work are summarized as follows: (i) The IVR propagator for SQ WP yields reasonably accurate results, particularly when the WP width dynamics are frozen or damped. (ii) By optimizing the WP width on the SQ potential, the accuracy of conventional HK FG scheme is significantly improved. (iii) With use of such optimized WP width, the HK FG and SQ schemes yield results of similar quality, which appears to come from the small difference between $S_{\mathrm{cl}}$ and $S_{\mathrm{sq}}^{\mathrm{sd}}$, as is particularly transparent in the SQ framework.

An essential part is that the IVR scheme connected consistently with the SQ WP is available. Hence, we shall run the conventional SQ WP MD simulation for equilibrium properties and incoherent processes, and turn on the IVR option in cases where more accurate QM treatment is required.

The (ii) above is supported also by a calculation on the potential employed in Refs. ${ }^{5}$ and $^{7}$ for which the optimization of $\rho$ on the SQ potential yields $\gamma=0.534$, in close agreement with the value 0.5 found optimal in Ref. ${ }^{7}$. Although this should be affirmed on more general theoretical ground, it sounds a natural presumption as it yields the optimal WP for the stationary ground state.

More assessments on various cases such as a double-well and many-dimensional systems yet remain to be done. Another issue to pursue is a combination with the electron WP theory ${ }^{39,40}$ where the WP width will play more critical roles. Works on these are now under way to be reported in due course.

\section{Acknowledgments}

The author acknowledges support from KAKENHI Nos. 20108017 (" $\pi$-space") and 22550012.
${ }^{1}$ E. J. Heller, J. Chem. Phys. 75 (1981) 2923.

2 W. H. Miller, J. Phys. Chem. A 105 (2001) 2942.

${ }^{3}$ M. Baranger, M. A. M. de Aguiar, F. Keck, H. J. Korsch, B. Schellhaaß, J. Phys. A: Math. Gen. 34 (2001) 7227.

${ }^{4}$ M. F. Herman, E. Kluk, Chem. Phys. 91 (1984) 27.

5 E. Kluk, M. F. Herman, H. L. Davis, J. Chem. Phys. 84 (1986) 326.

${ }^{6}$ M. F. Herman, J. Chem. Phys. 85 (1986) 2069.

${ }^{7}$ K. G. Kay, J. Chem. Phys. 100 (1994) 4377, 4432.

8 J. C. Burant, V. S. Batista, J. Chem. Phys. 116 (2002) 2748.

${ }^{9}$ C. Harabati, J. M. Rost, F. Grossmann, J. Chem. Phys. 120 (2004) 26.

10 A. Tanaka, Phys. Rev. A 73 (2006) 024101.

11 S. Koda, K. Takatsuka, Phys. Rev. A 83 (2011) 032117.
12 J. Tatchen, E. Pollak, G. Tao, W. H. Miller, J. Chem. Phys. 134 (2011) 134104.

13 P. A. J. Sherratt, D. V. Shalashilin, M. S. Child, Chem. Phys. 322 (2006) 127.

${ }^{14}$ H. D. Meyer, F. Gatti, G. A. Worth (Eds.), Multidimensional Quantum Dynamics: MCTDH Theory and Applications, WileyVCH, Weilheim, 2009.

15 A. Inoue-Ushiyama, K. Takatsuka, Phys. Rev. A 59 (1999) 3256.

${ }^{16}$ R. E. Wyatt, Quantum Dynamics with Trajectories, Springer, New York, 2005.

17 M. Tuckerman, B. J. Berne, G. J. Martyna, M. L. Klein, J. Chem. Phys. 99 (1993) 2796.

18 A. Nakayama, N. Makri, Proc. Nat. Acad. Sci. USA 102 (2005) 4230. 
19 J. A. Poulsen, G. Nyman, P. J. Rossky, Proc. Nat. Acad. Sci. USA $102(2005) 6709$.

20 J. Cao, G. A. Voth, J. Chem. Phys. 101 (1994) 6168.

${ }^{21}$ S. D. Ivanov, A. Witt, M. Shiga, D. Marx, J. Chem. Phys. 132 (2010) 031101.

22 S. Habershon, D. E. Manolopoulos, T. E. Markland, T. F. Miller III, Ann. Rev. Phys. Chem. 64 (2013) 387

${ }^{23}$ K. Ando, J. Chem. Phys. 121 (2004) 7136; Chem. Phys. Lett. 376 (2003) 532.

${ }^{24}$ N. Sakumichi, K. Ando, J. Chem. Phys. 128 (2008) 164516.

${ }^{25}$ K. Ando, J. Chem. Phys. 125 (2006) 014104; Phys. Rev. B 72 (2005) 172104.

${ }^{26}$ K. Hyeon-Deuk, K. Ando, J. Chem. Phys. 131 (2009) 064501; 132 (2010) 164507.

27 J. Ono, K. Ando, J. Chem. Phys. 137 (2012) 174503.

${ }^{28}$ J. Ono, K. Hyeon-Deuk, K. Ando, Int. J. Quant. Chem. 113 (2013) 356.

${ }^{29}$ K. Ando, J. T. Hynes, Adv. Chem. Phys. 110 (1999) 381.

${ }^{30}$ E. J. Heller, J. Chem. Phys. 62 (1975) 1544.

31 J. R. Klauder, Ann. Phys. 11 (1960) 123.

${ }^{32}$ H. Kuratsuji, Prog. Theor. Phys. 65 (1981) 224.
${ }^{33}$ H. G. Solari, J. Math. Phys. 27 (1986) 1351.

${ }^{34}$ F. Arickx, J. Broeckhove, E. Kesteloot, L. Lathouwers, P. van Leuven, Chem. Phys. Lett. 128 (1986) 310.

35 A. K. Pattanayak, W. C. Schieve, Phys. Rev. E 50 (1994) 3601.

${ }^{36}$ O. V. Prezhdo, Theor. Chem. Acc. 116 (2006) 206.

37 Y. Shigeta, Bull. Chem. Soc. Jpn. 82 (2009) 1323.

38 M. Hoshino, H. Nishizawa, H. Nakai, J. Chem. Phys. 135 (2011) 024111

39 K. Ando, Chem. Phys. Lett. 523 (2012) 134; Bull. Chem. Soc. Jpn. 82 (2009) 975.

40 K. Hyeon-Deuk, K. Ando, Chem. Phys. Lett. 532 (2012) 124.

${ }^{41}$ S. Zhang, E. Pollak, J. Chem. Phys. 119 (2003) 11058.

${ }_{42}$ M. F. Herman, A. Sergeev, J. Chem. Phys. 126 (2007) 034104.

43 A. L. Thompson, C. Punwong, T. J. Martinez, Chem. Phys. 370 (2010) 70

44 Y. Tsue, Prog. Theor. Phys. 88 (1992) 911.

${ }^{45}$ N. Watanabe, M. Tsukada, Phys. Rev. E 62 (2000) 2914.

${ }^{46}$ M. Suzuki, Phys. Lett. A 146 (1990) 319.

${ }^{47}$ D. F. Walls, G. J. Milburn, Quantum Optics, Springer, Berlin, 1994. 